\title{
LASS2 regulates invasion and chemoresistance via ERK/Drpl modulated mitochondrial dynamics in bladder cancer cells
}

\author{
Lijuan Huang, Ting Luan, Yujin Chen, Xin Bao, Yinglong Huang, Shi Fu, Haifeng Wang ${ }^{\bowtie}$, Jiansong Wang ${ }^{\bowtie}$ \\ Department of Urology, The Second Affiliated Hospital of Kunming Medical University, Yunnan Institute of Urology, Kunming 650101, China \\ $\triangle$ Corresponding authors: Haifeng Wang, Department of Urology, The Second Affiliated Hospital of Kunming Medical University, Yunnan Institute of \\ Urology, Kunming 650101, China. E-mail: highphone @ 126.com and Jiansong Wang, Department of Urology, The Second Affiliated Hospital of Kunming Medical \\ University, Yunnan Institute of Urology, Kunming 650101, China. E-mail: wangjiansong@kmmu.edu.cn \\ (C) Ivyspring International Publisher. This is an open access article distributed under the terms of the Creative Commons Attribution (CC BY-NC) license \\ (https://creativecommons.org/licenses/by-nc/4.0/). See http://ivyspring.com/terms for full terms and conditions.
}

Received: 2017.09.29; Accepted: 2018.01.28; Published: 2018.02.28

\begin{abstract}
Mitochondria coordinated a lot of vital cellular processes of energy production and distribution. Change of mitochondrial functions has been implicated in cancer progression. The present study aims to investigate the involvement of mitochondria dynamics in LASS2 induced invasion and chemoresistance of bladder cancer cells. $\mathrm{J} 82$ and BIU87 cell lines were used for LASS2 plasmid transfection while siRNA knockdown was carried out in 5637 cell line. Matrigel invasion assay and Annexin V/PI staining demonstrated that LASS2 negatively regulated cancer cell invasion and chemoresistance. JC-1 staining suggested that LASS2 overexpression downregulated mitochondrial membrane potential. Mitotracker staining showed that LASS2 induced mitochondrial fusion and inhibited mitochondrial fission. In addition, LASS2 overexpression downregulated expression of mitochondrial fission protein p-Drpl Drpl and Fis1. While depletion of LASS2 exhibited the opposite effects. Drpl inhibitor Mdivi abolished invasion and chemoresistance induced by LASS2 siRNA. Furthermore, we found that LASS2 overexpression could inhibit phosphorylation of ERK, which act upstream of Drpl. ERK inhibitor PD98059 suppressed Drpl phosphorylation and abrogated the effects of LASS2 depletion. In conclusion, the present study demonstrated that LASS2 inhibits bladder cancer invasion and chemoresistance through regulation of ERK-Drpl induced mitochondrial dynamics.
\end{abstract}

Key words: bladder cancer; LASS2; invasion; chemoresistance; mitochondria dynamics

\section{Introduction}

Bladder cancer is a common urological carcinoma and its incidence is rising in recent years [1]. Change of molecular markers influences the prognosis of bladder cancer [2-4]. Despite significant advances in therapies including surgery and chemotherapy in the past decade, poor survival of invasive bladder cancer made us to seek for more effective treatments. Thus it is important to clarify molecular mechanisms underlying the development of bladder cancer aggressiveness and drug resistance, which could be used for development of new therapy.

Downregulation of LASS2 has been found in various cancers including breast cancer [5], prostate cancer [6] and hepatocellular carcinoma [7]. LASS2 could enhance chemosensitivity of breast cancer through inhibition of V-ATPase proton pump activity and IAP signaling pathway [8]. Previously we reported that LASS2 negatively associated with poor clinical prognosis in bladder cancer patients [9]. LASS2 loss also leads to proliferation and invasion in bladder cancer cells [10]. Moreover, we demonstrated that LASS2 silenced enhances chemoresistance and V-ATPase activity in bladder cancer cells [11, 12]. A recent report demonstrated that liver-specific deletion of LASS2 delayed regeneration of mouse liver after partial hepatectomy with upregulation of cyclin A, 
$\mathrm{CDK} 2$ and $\mathrm{p}-\mathrm{Rb}$ [13]. In addition, combination of LASS2 and TGF- $\beta 1$ was an independent and significant risk factor for overall survival and time to recurrence in patients with hepatocellular carcinoma [14]. To date, the mechanism by which LASS2 attenuated bladder cancer invasion and chemoresistance has not been fully explored yet.

The mitochondrial system is highly evolved and controls energy production and distribution based on the availability of calories and oxygen[15]. Mitochondria play vital roles in many cellular processes including cell proliferation, metabolism and apoptosis. Mitochondrial dymamic is characterized by fission and fusion. Mounting evidence suggests that altered mitochondrial dynamics has been implicated in many human diseases. Altered balance of mitochondrial fission or fusion contributes to the development and progression of human cancers [16]. Recent reports suggested that cancer cells exhibited excess mitochondrial fission and impaired mitochondrial fusion due to an imbalance of Drp1/Mfn expression, which in turn influence cell cycle transition [17]. Additionally, it was reported that mitochondial dynamics play critical roles in metastasis of breast cancer[18].

In the present study, we linked the role of LASS2 with mitochondrial function. We demonstrated that LASS2 was able to induced mitochondrial fusion through inhibition of ERK/DRP1 signaling, which in turn reduces cancer invasion and chemoresistance.

\section{Materials and Methods}

\section{Cell culture and transfection}

Cancerous J82, T24, 5637, BIU-87 cell lines and normal uroepithelial SV-HUC-1, were obtained from American Type Culture Collection. Cells were cultured in DMEM medium (Gibco, USA) containing $10 \%$ FBS (Invitrogen, USA) at $37^{\circ} \mathrm{C}$ in $5 \% \mathrm{CO}_{2}$.

LASS2 siRNA and non-targeting siRNA were purchased from RiboBio (Guangzhou, China). The LASS2 siRNA sequence was 5'-AACCATCGTAAGA ATGACTGA-3'. LASS2 siRNA2 sequence is 5'-TGC GCTATAGGGTCACTTTAA-3'. LASS2 siRNA was transfected using DharmaFECT 1 Reagent (GE healthcare, CO, USA) according to the protocol. pCMV6LASS2 plasmid and pCMV6 empty plasmid were obtained from Origene (Origene, Rockville, USA). Lipofectamine 3000 transfection reagent was used for plasmid transfection (Invitrogen, USA).

\section{Quantitative real-time PCR}

RNA was extracted using Trizol (Life technology, USA). Reverse transcription was performed using PrimeScript RT Master Mix (TAKARA). PCR was carried out using ABI 7500 PCR system (Life technology, USA). The relative level of target gene expression was calculated by the $2^{-\Delta \Delta C t}$ method.

\section{Western blot}

Total protein was extracted by lysis buffer and quantified by Bradford method. About $50 \mu \mathrm{g}$ protein was transferred to PVDF membranes after separated by SDS-PAGE. The membranes were incubated at $4^{\circ} \mathrm{C}$ overnight with primary antibodies against LASS2 (1:800, Proteintech, USA), Drp1, p-Drp1, Fis1, p-ERK, ERK, cleaved caspase 3, cytochrome c (1:1000, Cell Signaling Technology, Boston, MA, USA) and GAPDH (1:3000, Cell Signaling Technology, Boston, MA, USA). After incubation with HRP-coupled secondary antibodies (1:2000) at room temperature for 2 hours, ECL (Pierce, Rockford, IL, USA) was used to visualize proteins by DNR BioImaging System (DNR, Israel).

\section{Matrigel invasion assay}

Cell invasion assay was performed using a transwell chamber coated with Matrigel from BD Bioscience. Cells were suspended in $100 \mu \mathrm{l}$ of serum-free medium and transferred to the upper chamber and incubated for 20 hours. Medium with $15 \%$ FBS was added to the lower chamber. The non-invading cells in the upper chambers were removed by a cotton tip. Cells passed through the filter were fixed, stained with hematoxylin and counted under microscope.

\section{CCK8 assay}

CCK8 assay was performed to detect relative cell viability using Cell Counting Kit-8 kit from Dojindo (Dojindo, Gaithersburg, MD) according to the manufacturer's protocol. Briefly, cultured cells were incubated with $10 \mu \mathrm{l} /$ well of CCK-8 solution for 4 hours in the incubator. Then each well was measured at the wave length of $490 \mathrm{~nm}$.

\section{Annexin V/PI staining}

For determination of apoptosis, cells were stained with Annexin V/PI kit (BD bioscience) and measured by BD FACS Calibur flow cytometer (Becton Dickinson, USA).

\section{JC-1 staining (Mitochondrial membrane potential)}

The mitochondrial membrane potential $(\Delta \psi \mathrm{m})$ was analyzed by JC-1 staining kit from Cell Signaling Technology. Cells were analyzed using BD FACS Calibur flow cytometer (Becton Dickinson, USA).

\section{Immunofluorescence}

Mitochondria in cancer cells was stained using MitoTracker red (Life Technologies, USA) for 30 minutes. Images were taken by BX53 Olympus micro- 
scope. Mean mitochondrial length was determined by measuring 10 individual mitochondria from cells obtained by fluorescence microscopy using cellSens software (Olympus)

\section{Statistical analysis}

SPSS 17.0 was used for analysis. t-test was used to compare data. $\mathrm{p}<0.05$ was considered to be statistical significant.

\section{Results}

\section{LASS2 overexpression inhibits invasion and chemoresistance of bladder cancer cells.}

We examined the expression of LASS2 protein expression in normal uroepithelial cell line SV-HUC-1 and 4 bladder cancer cell lines (BIU87, J82, 5637 and T24). As shown in Figure 1A, 5637 showed the relative higher LASS2 expression. LASS2 expression in normal SV-HUC-1 was higher than the other 3 cell lines. We transfected LASS2 plasmid in J82 and BIU87 cells and depleted LASS2 in 5637 cells by siRNA. The transfection efficiency was confirmed as shown in Figure 1B. The effects of LASS2 overexpression has not been validated before in bladder cancer cells. Then we examined the change of invasion and chemoresistance. Matrigel invasion assay was used to examine the change of invading ability. We found that forced expression of LASS2 significantly decreased invading cell number in both BIU87 and J82 cells. Accordingly, LASS2 depletion in 5637 cells facilitated cell invasion (Figure 2A).

$1 \mu \mathrm{M}$ doxorubicin (DOX) was used to treat bladder cancer cells. CCK-8 analysis and Annexin $\mathrm{V} / \mathrm{PI}$ staining were used to check the change of cell viability and apoptosis. As shown in Figure 2B, LASS2 overexpression impaired cell viability of J82 and BIU87 cells treated with doxorubicin for 24 and 48 hours. In contrast, LASS2 depletion in 5637 cells enhanced cell viability after DOX treatment. Annexin V/PI staining demonstrated that after 24 hours DOX treatment, the rates of apoptosis in BIU87 and J82 cells were significantly increased after LASS2 overexpression (Figure 2C). While the rate of apoptosis in 5637 cells transfected with LASS2 siRNA was lower than that of control cells. In addition, we found that cytochrome c and cleaved caspase 3 were significant upregulated after LASS2 overexpression while its depletion showed the opposite effects (Figure S1). These results above demonstrated LASS2 overexpression inhibits invasion and chemoresistance in bladder cancer cells.

\section{LASS2 regulates mitochondrial dynamics and membrane potential in bladder cancer cells.}

Resistance to chemotherapy is frequently regulated by mitochondrial apoptosis pathway. Then we explored if LASS2 could control mitochondrial apoptosis by regulating mitochondrial membrane potential $(\Delta \psi \mathrm{m})$. We carried out JC-1 staining using flow cytometry. In cells with low membrane potential, JC-1 showed green fluorescence instead of red. We found that in J82 and BIU87 with LASS2 transfection, the percentage of red fluorescence decreased compared with control, indicating decreased mitochondrial membrane potential. While in 5637 cells with LASS2 siRNA, the percentage of red fluorescence was upregulated (Figure 3A). These data indicated that LASS2 is a negative regulator of mitochondrial membrane potential. To find out if LASS2 is involved in the mitochondrial dynamic balance, we checked mitochondrial morphology. Mitochondria in cells with LASS2 overexpression was more prone to fusion with elongated mitochondrial shape in BIU87 and J82 cells. While in 5637 cells transfected with LASS2 siRNA, mitochondria had increased fission with increased punctuate mitochondria and decreased mitochondrial length (Figure 3B). These results demonstrated that LASS2 was able to induce mitochondrial fusion and inhibit $\Delta \psi \mathrm{m}$, which sensitizes bladder cancer cells to mitochondrial apoptosis.

A

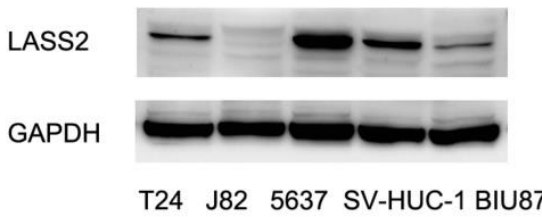

B
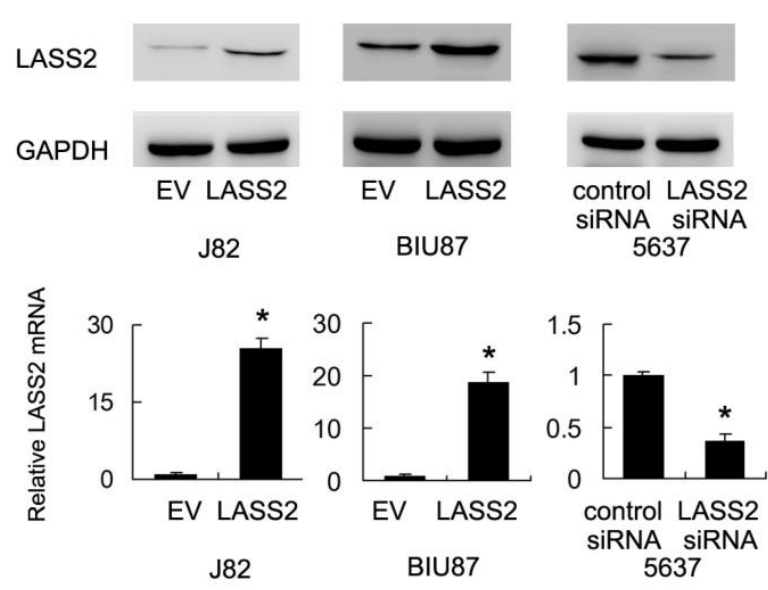

Figure 1. Expression and transfection efficiency of LASS2 in bladder cancer cells A. Relative expression of LASS2 protein expression in normal uroepithelial cell line SV-HUC-1 and 4 bladder cancer cell lines (BIU87, J82, 5637 and T24). B. Plasmid transfection of LASS2 significant upregulated its protein and mRAN expression in both BIU87 and $\mathrm{J} 82$ cell lines. siRNA knockdown reduced its expression in 5637 cell line. *: $\mathrm{p}<0.05$. 
A J82

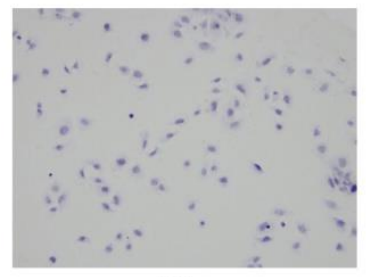

EV

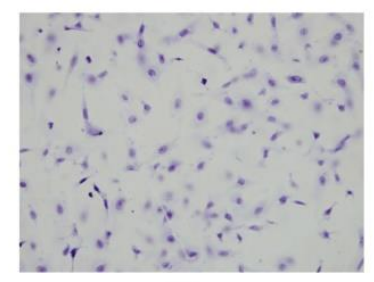

EV

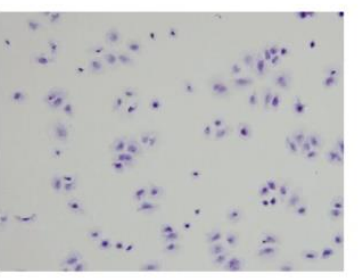

Control siRNA

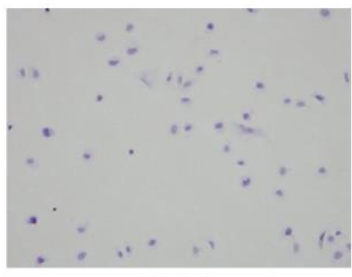

LASS2

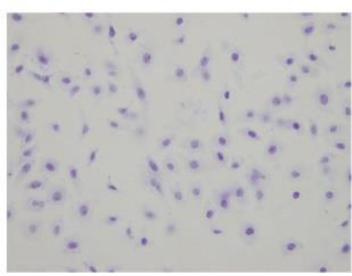

LASS2

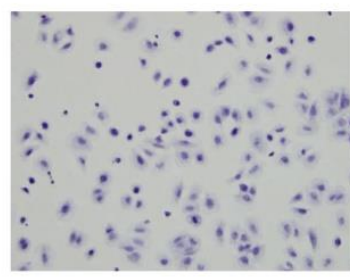

LASS2 SiRNA
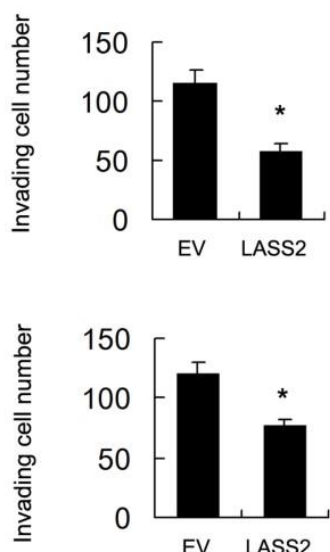

EV LASS2

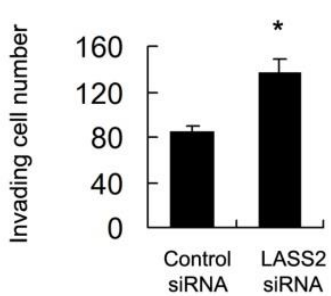

B

C
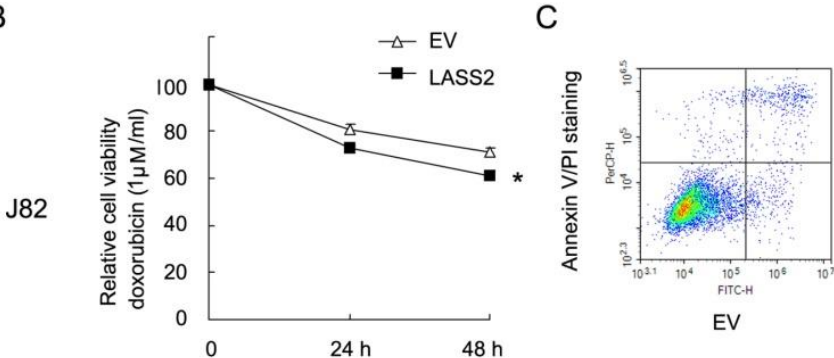

EV

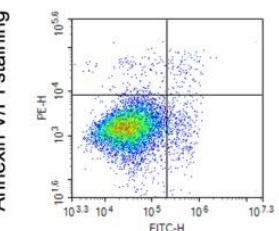

EV

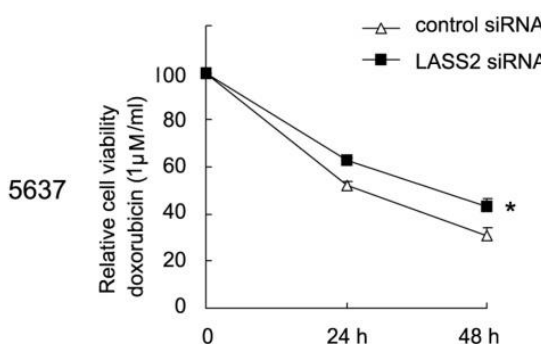

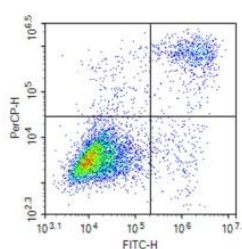

LASS2
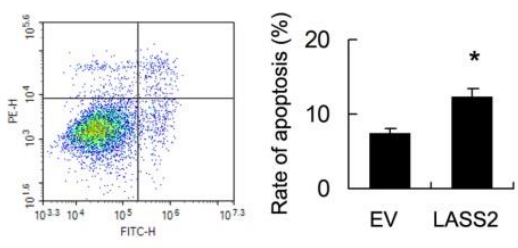

LASS2
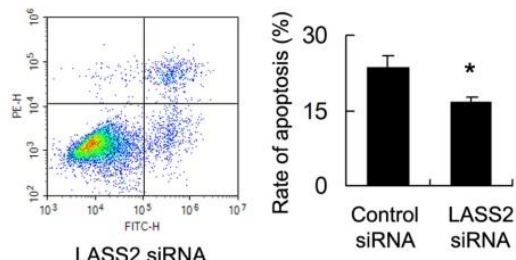

Figure 2. LASS2 overexpression inhibits invasion, chemoresistance of bladder cancer cells. A. Matrigel invasion assay demonstrated that transfection of LASS2 plasmid significantly downregulated invading cell number in both BIU87 and 382 cell line. LASS2 siRNA depletion in 5637 cell line upregulated the number of invading cells. B. CCK-8 analysis showed that LASS2 overexpression downregulated cell viability in J82 and BIU87 cell line treated with $1 \mu$ M doxorubicin for 24 and 48 hours. LASS2 depletion in 5637 cells upregulated the level of cell viability after $1 \mu \mathrm{M}$ doxorubicin treatment. C. Annexin V/PI staining showed that after 24 hours doxorubicin treatment, the rates of apoptosis in BIU87 and $\mathbf{8 2} 2$ cells were significantly increased after LASS2 overexpression. LASS2 siRNA downregulated the rate of apoptosis in 5637 cells. *: $\mathrm{p}<0.05$. 
A J82
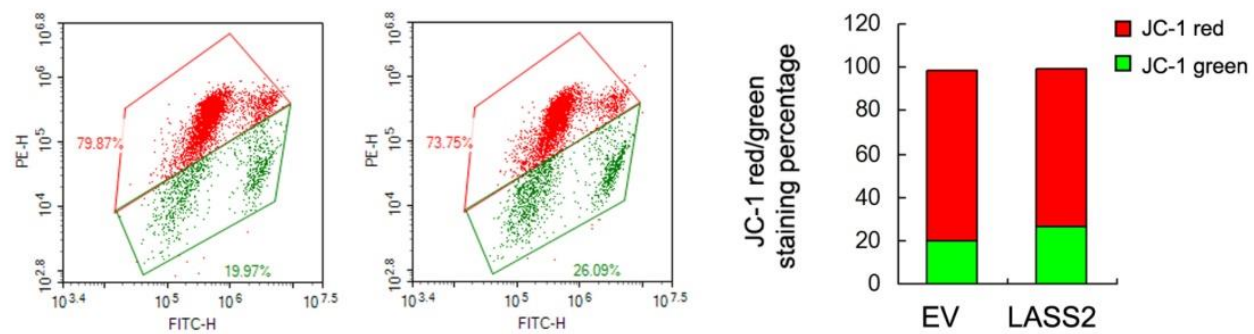

EV
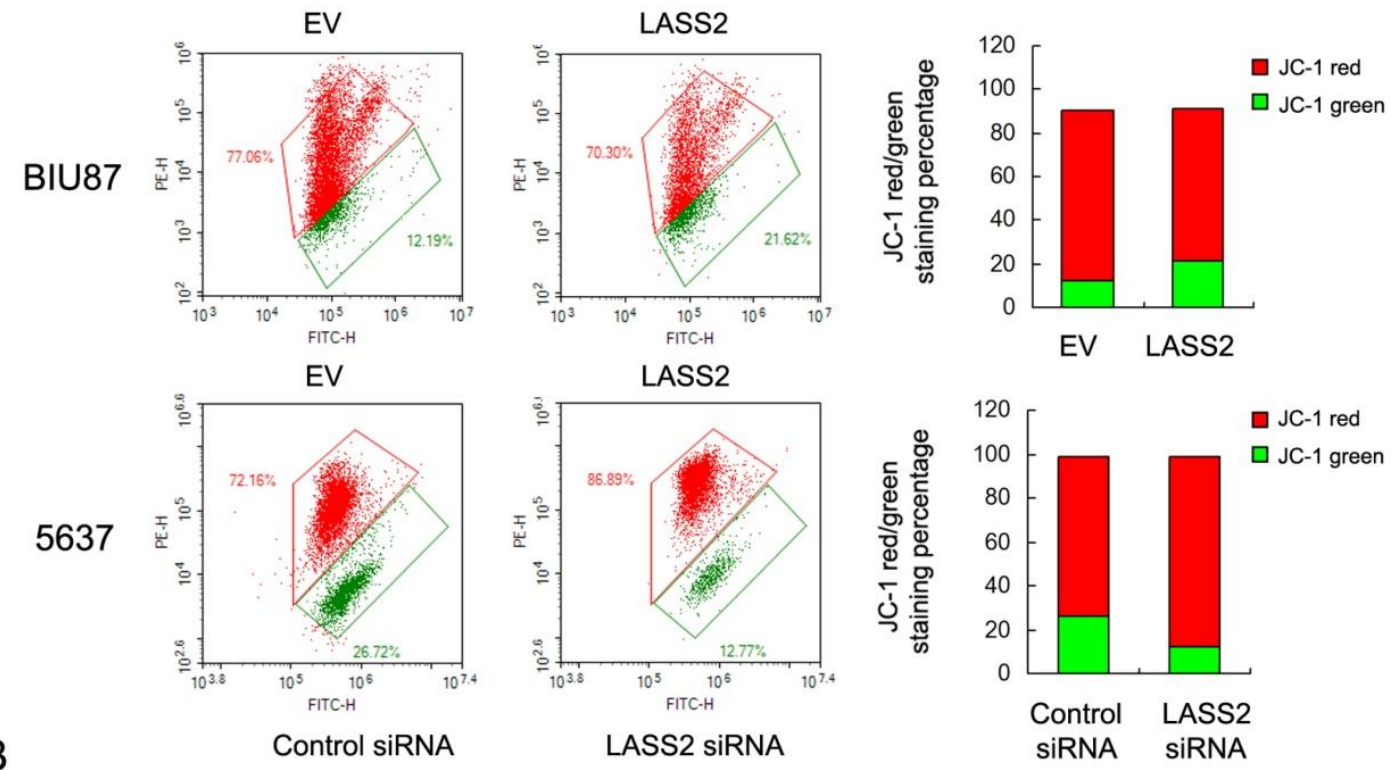

J82

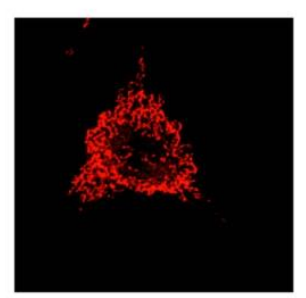

EV

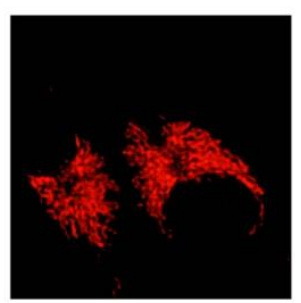

EV

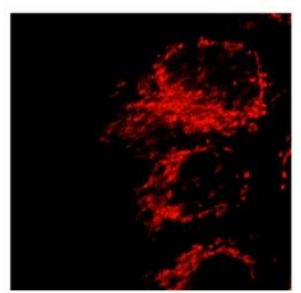

Control siRNA

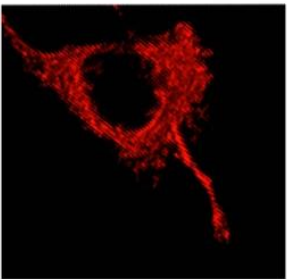

LASS2

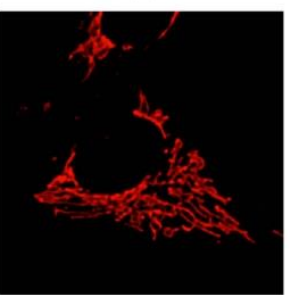

LASS2

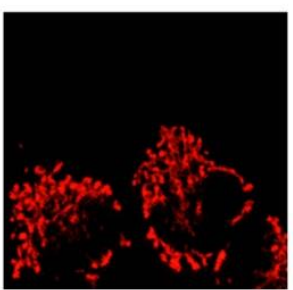

LASS2 SiRNA

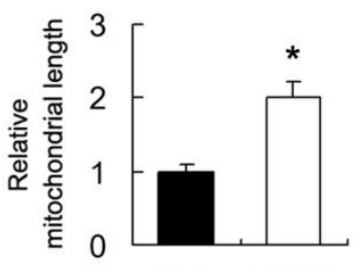

EV LASS2

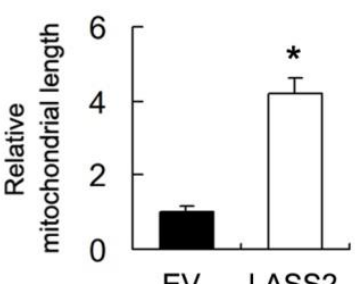

EV LASS2

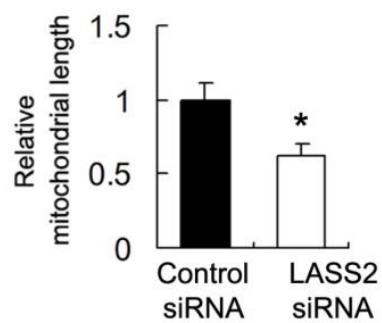

Figure 3. LASS2 regulates mitochondrial dynamics and membrane potential in bladder cancer cells. A. Flow cytometry/JC-1 staining showed that in 182 and BIU87, LASS2 transfection downregulated the percentage of red fluorescence. In 5637 cells, LASS2 siRNA upregulated the percentage of red fluorescence. B. Mitrotracker Red staining showed that LASS2 transfection increase the level of mitochondria length (fusion) in J82 and BIU87 cells. While in 5637 cells LASS2 depletion decreased mitochondria length (fission). *: $\mathrm{p}<0.05$. 


\section{LASS2 inhibits Drp 1 signaling}

To investigate the mechanisms underlying mitochondrial function regulated by LASS2, we examine proteins that directly regulate mitochondrial dynamics including p-Drp1, Drp1 and Fis1. Upregulation of p-Drp1 and Fis1 induces mitochondrial fission, which has been shown in inducing cancer cell invasion. As shown in Figure 4, we demonstrated that LASS2 overexpression downregulated the expression of p-Drp1 as well as Drp1, Fis1 in BIU87 and J82 cells. While LASS2 depletion upregulated these proteins.

\section{The role of LASS2 in bladder cancer invasion and chemoresistance was partly dependent on Drp I signaling}

To validate the involvement of mitochondrial fission/fusion balance and Drp1 signaling in LASS2 controlled invasion and chemoresistance, we adopted Mdivi-1, a specific blocker of Drp1 signaling, to treat 5637 cells. As shown in Figure 5A, Mdivi-1 treatment induced mitochondrial fusion, which reversed the role of LASS2 siRNA. We also found that Mdivi-1 reduced invading cell number and upregulated the percentage of apoptosis, abrogated the biological effects of LASS2 siRNA in 5637 cells (Figure 5B), which validated the involvement of Drp1/mitochondrial dynamics.

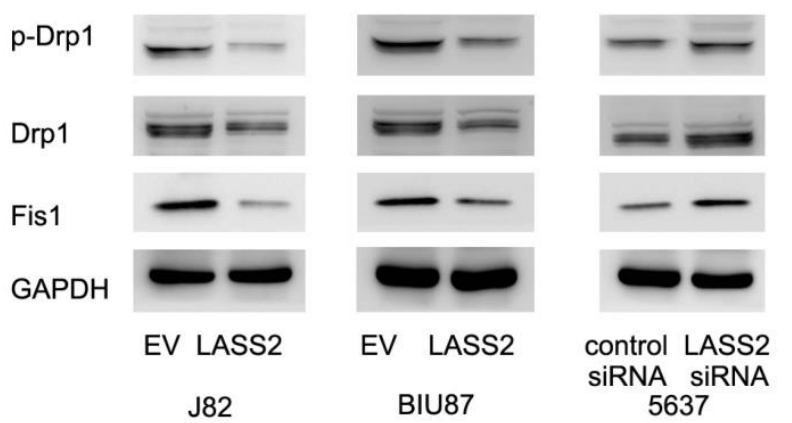

Figure 4. LASS2 inhibits Drpl signaling in bladder cancer cells. LASS2 overexpression downregulated the expression of p-Drpl, Drpl and Fis1 in 182 and BIU87 cell lines. LASS2 depletion in 5637 cell line upregulated the expression of $\mathrm{p}$-Drpl, Drpl and Fis1.

A

5637

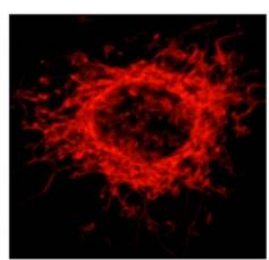

Control siRNA

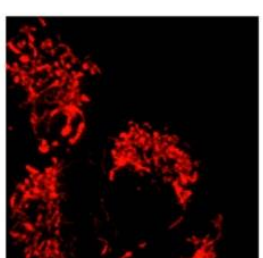

LASS2 SiRNA

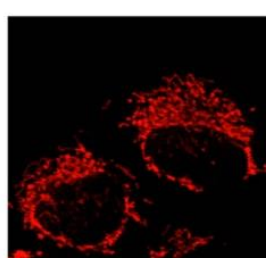

LASS2 SIRNA Mdivi-1

B 5637

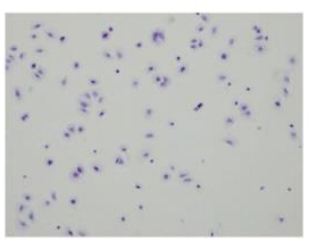

Control siRNA

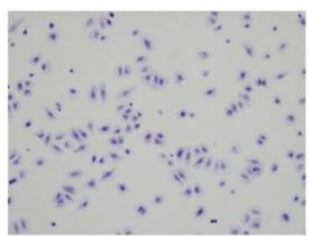

LASS2 SiRNA

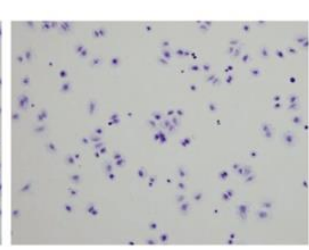

LASS2 SIRNA Mdivi-1

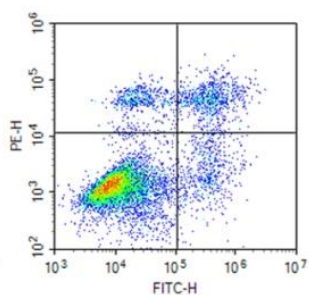

LASS2 SIRNA Mdivi-1

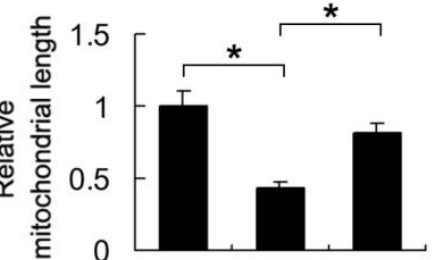

Control LASS2 LASS2 SIRNA SiRNA SIRNA Mdivi-1

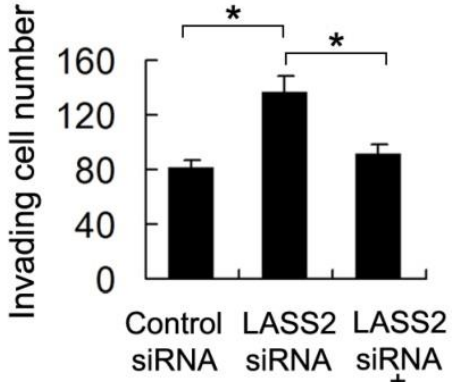

Mdivi-1

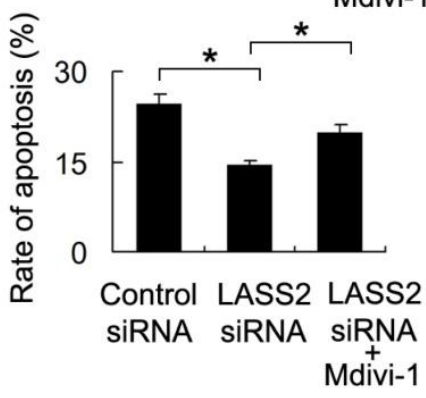

Figure 5. Drp 1 inhibitor reduces the effect of LASS2 depletion on invasion and chemoresistance. A. Mitochondrial morphology was evaluated by Mitotracker Red staining after treatment with Mdivi-1. Mdivi-1 treatment induced mitochondrial fusion and reversed fission induced by LASS2 depletion. B. Mdivi-1 reduced invading cell number and upregulated the percentage of apoptosis, reversing the effect of LASS2 depletion in 5637 cells. *: p<0.05. 


\section{LASS2 inhibits Drp 1 signaling through regulation of ERK pathway.}

To further examine the possible mechanism of LASS2 suppressed Drp1 signaling. We profiled the expression of established pathways related with invasion and chemoresistance. Previous studies have reported that Drp1 was a downstream target of ERK signal pathway, which could phosphorylated Drp1 at S616. Here, we found that LASS2 was able to inhibit ERK phosphorylation and LASS2 depletion upregulated ERK phosphorylation. Treatment with MEK/ERK inhibitor PD98059 in 5637 cells abolished the effect of LASS2 depletion on p-Drp1(Figure 6). To exclude the off-target effect of siRNA. We also used a second siRNA to repeat the above findings. As shown in Figure S2, LASS2 siRNA2 upregulated p-Drp1 and p-ERK signaling in 5637 cell line.
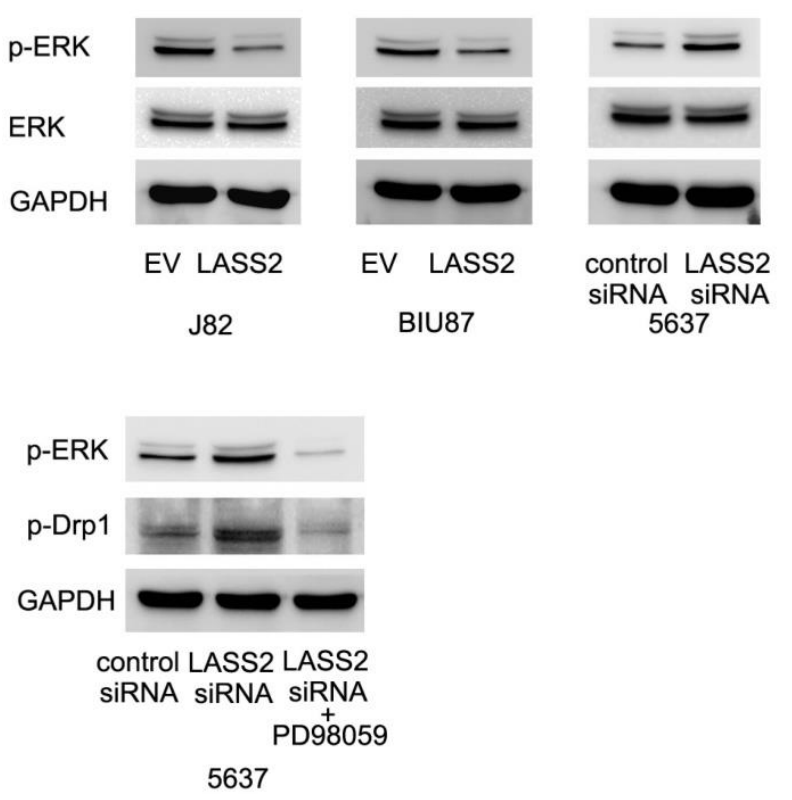

Figure 6. LASS2 inhibits Drp1 signaling through regulation of ERK pathway. LASS2 overexpression inhibited ERK phosphorylation in BIU87 and j82 cells while LASS2 depletion induced ERK phosphorylation in 5637 cells. Treatment with ERK inhibitor PD98059 in 5637 cells reduced p-Drpl expression, abolishing the effect of LASS2 siRNA.

\section{Discussion}

Growing evidences suggest LASS2 as an important tumor suppressor downregulated in various cancers including breast [5], prostate [6] and liver cancer [7]. Our previous study reported that LASS2 negatively associated with poor clinical prognosis in bladder cancer patients [9]. Functional study was carried by LASS2 siRNA. We showed that its loss leads to proliferation and invasion, with elevated chemoresistance and V-ATPase activity in bladder cancer cells[10-12]. However, the mechanism by which LASS2 affects bladder cancer invasion and chemoresistance has not been fully identified yet.

In this study, we validated the role of LASS2 overexpression in bladder cancer cell lines, which has not been performed before. As expected, LASS2 transfection inhibited invasion and Doxorubicin (DOX) resistance in bladder cancer cells. We also validated this function by using LASS2 siRNA, which showed the opposite effects. These data further confirmed the biological function of LASS2 as a tumor suppressor in bladder cancer cell lines. Chemotherapeutic drug could induce apoptosis by mitochondrial pathway. So we checked if LASS2 was able to influence mitochondrial apoptosis by changing mitochondrial function, which includes mitochondrial dynamics and mitochondrial membrane potential. Accordingly, JC-1 staining demonstrated that LASS2 was able to suppress mitochondrial membrane potential. It was reported that loss of membrane potential induces mitochondrial apoptosis pathway through increased mitochondrial membrane permeability and release of cytochrome c, which in turn activate apoptosis cascade $[19,20]$.

Mitochondrial dynamics (fusion and fission balance) could be regulated by the level of Drp1 phosphorylation [21], which play an important role during metabolic control of cancer cells. To further explore the role of LASS2 in mitochondrial function and metabolism, we stained bladder cancer cells with Mitotracker. The results indicated that LASS2 overexpression downregulated mitochondrial fission while upregulated fusion, leading to elongated mitochondrial shape. Accordingly, western blot showed that LASS2 downregulated p-Drp1 and Fis1 protein expression.

It was reported that mitochondrial fission could lead to cancer cell migration and invasion [22]. While mitochondrial fusion suppresses cell migration and invasion in vitro and in vivo [23]. Inhibition of mitochondrial fission by Drp1 depletion or inhibitor blocked cell cycle and resulted in apoptosis in lung and colon cancer cells [17, 24]. Mitochondrial fission has been shown to repress respiratory complex I and IV. Since respiratory complex I and -III are the main sources of ROS, repression of complex I by fission provides an explanation for resistance to ROS induced mitochondrial apoptosis $[25,26]$. Thus it is possible LASS2 inhibit bladder cancer invasion and chemoresistance through induction of mitochondrial fusion.

Previous studies showed that LASS2 could inhibit V-ATPase activity [27-29]. A recent report suggested that V-ATPase depletion affects mitochondrial ATPase function [30]. V-ATPase function has also been linked to cellular aging and rejuvenation by controlling mitochondrial function [31], suggesting 
that LASS2 may affect mitochondrial function partly through V-ATPase regulation. In addition, since the $\mathrm{V}$-ATPase and the mitochondrial ATPase are structurally and mechanistically related. It is possible that LASS2 could also inhibit mitochondrial ATPase activity as well, which needs further investigation.

In this study, we also demonstrated that ERK inhibition is partly responsible for the downregulation of Drp1 signaling. ERK is well-known for its role in cancer invasion and apoptosis. ERK could activate Drp1 phosphorylation during reprogramming of stem cell to pluripotency [32]. It is reported that bone marrow-derived mesenchymal stem cells activates ERK signaling, which in turn phosphorylated pro-fission factor Drp1 at residue S616 and promotes cancer chemoresistance [33]. These reports support our hypothesis that LASS2 inhibits Drp1 signaling and chemoresistance through blockage of ERK pathway.

In conclusion, this study demonstrated that LASS2, a potential tumor suppressor, influences bladder cancer invasion and chemoresistance through, at least partly, ERK/Drp1 regulated mitochondrial fission/fusion balance.

\section{Supplementary Material}

Supplementary figures.

http://www.jcancer.org/v09p1017s1.pdf

\section{Acknowledgements}

This work was supported by the National Natural Science Foundation of China (No. 81660422, No. 81660423, No. 81460384), Joint Project of Science and Technology, Department of Yunnan and Kunming Medical University (No. 2014FA015, No. 2014FZ031), and Project of Yunnan Provincial Science and Technology (No. 2015FB196).

\section{Competing Interests}

The authors have declared that no competing interest exists.

\section{References}

1. Siegel R, Ma J, Zou Z, et al. Cancer statistics. CA: Cancer J Clin. 2014; 64(1): 9-29.

2. Reddy O L, Cates J M, Gellert L L, et al. Loss of FOXA1 Drives Sexually Dimorphic Changes in Urothelial Differentiation and Is an Independent Predictor of Poor Prognosis in Bladder Cancer. Am J Pathol. 2015; 185(5): 1385-95.

3. Szarvas T, Laszlo V, Vom Dorp F, et al. Serum endostatin levels correlate with enhanced extracellular matrix degradation and poor patients' prognosis in bladder cancer. Int J Cancer. 2012; 130(12): 2922-9.

4. Yang G L, Zhang L H, Bo J J, et al. Increased expression of HMGB1 is associated with poor prognosis in human bladder cancer . J Surg Oncol. 2012; 106(1): 57-61.

5. Mei F, You J, Liu B, et al. LASS2/TMSG1 inhibits growth and invasion of breast cancer cell in vitro through regulation of vacuolar ATPase activity. Tumour Biol. 2015; 36(4):2831-44

6. Xu X, Liu B, Zou P, et al. Silencing of LASS2/TMSG1 enhances invasion and metastasis capacity of prostate cancer cell . J Cell Biochem. 2014; 115(4): 731-43.
7. Chen $\mathrm{L}$, Lu X, Zeng $\mathrm{T}$, et al. Enhancement of DEN-induced liver tumourigenesis in hepatocyte-specific Lass2-knockout mice coincident with upregulation of the TGF-beta1-Smad4-PAI-1 axis . Oncol Rep. 2014; 31(2): 885-93.

8. Sun C, Li N, Yang Z, et al. miR-9 regulation of BRCA1 and ovarian cancer sensitivity to cisplatin and PARP inhibition . J Natl Cancer Inst. 2013; 105(22): $1750-8$

9. Wang $\mathrm{H}$, Wang J, Zuo $\mathrm{Y}$, et al. Expression and prognostic significance of a new tumor metastasis suppressor gene LASS2 in human bladder carcinoma . Med Oncol. 2012; 29(3): 1921-7.

10. Zhao Q, Wang H, Yang M, et al. Expression of a tumor-associated gene, LASS2, in the human bladder carcinoma cell lines BIU-87, T24, EJ and EJ-M3. Exp Ther Med. 2013; 5(3): 942-946.

11. Wang $\mathrm{H}$, Zuo $\mathrm{Y}$, Ding $\mathrm{M}$, et al. LASS2 inhibits growth and invasion of bladder cancer by regulating ATPase activity. Oncol Lett. 2017; 13(2): 661-668.

12. Wang $\mathrm{H}$, Zhang $\mathrm{W}$, Zuo $\mathrm{Y}$, et al. miR-9 promotes cell proliferation and inhibits apoptosis by targeting LASS2 in bladder cancer. Tumour Biol. 2015; 36(12): $9631-40$.

13. Jin H, Wang C, Gu D, Zhang Y, Fan S, Xing S, et al. Liver-specific deletion of LASS2 delayed regeneration of mouse liver after partial hepatectomy. Biochemical and biophysical research communications. 2017; 493: 1176-83.

14. Ruan H, Wang T, Yang C, Jin G, Gu D, Deng X, et al. Co-expression of LASS2 and TGF-beta1 predicts poor prognosis in hepatocellular carcinoma. Scientific reports. 2016; 6: 32421.

15. Zong W X, Rabinowitz J D, and White E. Mitochondria and Cancer. Mol Cell. 2016; 61(5): 667-676.

16. Archer S L. Mitochondrial dynamics--mitochondrial fission and fusion in human diseases. N Engl J Med. 2013; 369(23): 2236-51.

17. Rehman J, Zhang $\mathrm{H} \mathrm{J}$, Toth $\mathrm{P} \mathrm{T}$, et al. Inhibition of mitochondrial fission prevents cell cycle progression in lung cancer. FASEB J. 2012; 26(5): 2175-86.

18. Zhao J, Zhang J, Yu M, et al. Mitochondrial dynamics regulates migration and invasion of breast cancer cells. Oncogene. 2013; 32(40): 4814-24.

19. Chen X, Wong J Y, Wong $\mathrm{P}$, et al. Low-dose valproic acid enhances radiosensitivity of prostate cancer through acetylated p53-dependent modulation of mitochondrial membrane potential and apoptosis . Mol Cancer Res. 2011; 9(4): 448-61.

20. Hong J, Samudio I, Chintharlapalli S, et al. 1,1-bis(3'-indolyl)-1-(p-substituted phenyl)methanes decrease mitochondrial membrane potential and induce apoptosis in endometrial and other cancer cell lines . Mol Carcinog. 2008; 47(7): 492-507.

21. Chan D C. Mitochondrial fusion and fission in mammals. Annu Rev Cell Dev Biol. 2006: 22. 79-99.

22. Ferreira-da-Silva A, Valacca C, Rios E, et al. Mitochondrial dynamics protein Drp1 is overexpressed in oncocytic thyroid tumors and regulates cancer cell migration . PLoS One. 2015; 10(3): e0122308.

23. Zhang B, Wang J, Huang Z, et al. Aberrantly upregulated TRAP1 is required for tumorigenesis of breast cancer. Oncotarget. 2015; 6(42): 44495-508.

24. Inoue-Yamauchi A and Oda H. Depletion of mitochondrial fission factor DRP1 causes increased apoptosis in human colon cancer cells. Biochem Biophys Res Commun. 2012; 421(1): 81-5.

25. Muliyil S and Narasimha M. Mitochondrial ROS regulates cytoskeletal and mitochondrial remodeling to tune cell and tissue dynamics in a model for wound healing. Dev Cell. 2014; 28(3): 239-52.

26. Park J, Lee J, and Choi C. Mitochondrial network determines intracellular ROS dynamics and sensitivity to oxidative stress through switching inter-mitochondrial messengers. PLoS One. 2011; 6(8): e23211.

27. Gu D, Jin H, Jin $G$, et al. The asialoglycoprotein receptor suppresses the metastasis of hepatocellular carcinoma via LASS2-mediated inhibition of V-ATPase activity. Cancer letters. 2016; 379: 107-16.

28. Fan SH, Wang YY, Wu ZY, et al. AGPAT9 suppresses cell growth, invasion and metastasis by counteracting acidic tumor microenvironment through KLF4/LASS2/V-ATPase signaling pathway in breast cancer. Oncotarget. 2015; 6: 18406-17.

29. Fan S, Niu Y, Tan N, et al. LASS2 enhances chemosensitivity of breast cancer by counteracting acidic tumor microenvironment through inhibiting activity of V-ATPase proton pump. Oncogene. 2013; 32: 1682-90.

30. Baker N, Hamilton G, Wilkes JM, et al. Vacuolar ATPase depletion affects mitochondrial ATPase function, kinetoplast dependency, and drug sensitivity in trypanosomes. Proceedings of the National Academy of Sciences of the United States of America. 2015; 112: 9112-7.

31. Hughes $\mathrm{AL}$, Gottschling DE. An early age increase in vacuolar $\mathrm{pH}$ limits mitochondrial function and lifespan in yeast. Nature. 2012; 492: 261-5.

32. Prieto J, Leon M, Ponsoda X, et al. Early ERK1/2 activation promotes DRP1-dependent mitochondrial fission necessary for cell reprogramming. Nat Commun. 2016; 7: 11124

33. Cai J, Wang J, Huang $Y$, et al. ERK/Drp1-dependent mitochondrial fission is involved in the MSC-induced drug resistance of T-cell acute lymphoblastic leukemia cells. Cell Death Dis. 2016; 7(11): e2459. 